\title{
Prehabilitation for lymphedema in head and neck cancer patients at a community cancer center
}

\author{
Andrew Sember, BA; Cheryl Pranskevich, PT, CLT; Susan T Scott, BSN, RN, OCN; Ian V \\ Hutchinson, PhD, DSc; and Rex Hoffman, MD \\ Disney Family Cancer Center, Providence St Joseph Medical Center, Burbank, California
}

Patients with head and neck cancer often develop morbidities as a result of their treatment with surgery, radiation, and chemotherapy. One of the most prevalent side effects of the treatment is lymphedema, the accumulation of interstitial fluid in tissues that have inadequate lymph drainage. Secondary lymphedema, an acquired abnormality in the lymphatic network, is commonly caused by cancer and/or its treatment. Lymphedema is both under-recognized and under-treated in head and neck cancer. While recent advances in radiation therapy techniques have resulted in a corresponding drop in other treatment-related morbidities, an estimated $50 \%$ of treated head and neck cancer patients will develop lymphedema. Indeed, at some places the incidence is much higher, at $75 \%$, following treatment with surgery and radiation. Clearly, there is an unmet need to recognize and treat lymphedema in head and neck cancer patients. This article describes an early intervention prehabilitation program that was established for the early identification and treatment of patients at risk of lymphedema and compares the observed outcomes before and after the initiation of the program.

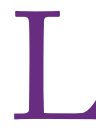

ymphedema is the swelling of tissue caused by the accumulation of interstitial fluid in any area of the body where lymphatic flow has been compromised. ${ }^{1}$ Secondary lymphedema is an acquired abnormality in lymph drainage ${ }^{1,2}$ and is the type commonly seen in cancer patients. Secondary lymphedema can be described as external or internal. Internal lymphedema, swelling of deep structures and tissues, is very difficult to quantify.

Lymphedema in patients with head and neck cancers

Lymphedema is a complicating morbidity frequently seen in head and neck cancer patients who have undergone treatment with surgery, radiation, and chemotherapy. However, although it is one of the most prevalent side effects of treatment, it is both under-recognized and under-treated. ${ }^{3}$

In head and neck cancer patients, internal swelling may develop in the soft tissues of the upper aerodigestive tract, ${ }^{4}$ affecting articulation and swallowing. Currently, there does not seem to be an effective practical and reliable tool with which to measure internal lymphedema. In addition, it is generally accepted that there is no effective way to treat internal lymphedema. By contrast, external lymphedema is more readily observed, but both subjective and objective assessments are difficult. External swelling may occur in the face, jaw, and neck. However, the subjective scales currently available are insufficient to capture very important characteristics of external lymphedema. ${ }^{5}$ The Edge Task Force on Head and Neck Cancer in 2015 was not able to recommend any outcome measures for objectively quantifying external edema. ${ }^{6}$ Furthermore, objective measurements of head and neck lymphedema can be expensive and time consuming.

\section{Extent and risk}

A combination of both internal and external swelling is seen in more than $50 \%$ of patients. ${ }^{7}$ Risk factors include "throat" tumors, multicancer treatment approaches, higher total radiation dose, a greater number of radiation procedures, and radiation at the surgical site. ${ }^{5}$ More than 500,000 survivors of head and neck cancer in the United States are at risk of lymphedema. ${ }^{5}$ Although recent advances in treatment have reduced the incidence of other morbidities, $50 \%$ of patients who are treated for head and neck cancer may still develop lymphedema. ${ }^{1,8}$ The reported incidence in some centers may be much higher, with up to $75 \%$ of patients developing lymphedema following treatment. ${ }^{9}$

Accepted for publication April 21, 2017. Correspondence: Ian V Hutchinson, PhD, DSc: lan.Hutchinson@providence. org. Disclosures: The authors report no disclosures or conflicts of interest. JCSO. 2017;15(3):e127-e134. (O2017 Frontline Medical Communications. doi: https://doi.org/10.12788/jcso.0345 


\section{Measurement modalities for clinical evaluation}

There is little current research into lymphedema of the head and neck, despite the high prevalence of the condition. ${ }^{8}$ According to Deng and colleagues, measurement of head and neck lymphedema is a challenge, which has an impact on clinical assessment, diagnosis, and treatment of this under-recognized, under-reported and underaddressed problem in head and neck cancer patients. ${ }^{10}$ In a review of the literature, Deng and colleagues identified three measurement modalities available for clinical evaluation: patient-reported outcomes, clinician-reported outcomes, and technology. ${ }^{10}$ One major factor, though, in detecting lymphedema, is physician awareness: physicians, health care professionals, and even some lymphedema therapists are not well educated about this problem. ${ }^{8}$

\section{Treatment}

The effectiveness of traditional lymphedema treatment is not well defined. ${ }^{8}$ Currently, complete decongestive therapy (CDT), is considered the standard of care for lymphedema. The National Lymphedema Network has stated that modifications of CDT, especially manual lymphatic drainage and modified compressive garments for external lymphedema, have been shown to be beneficial for the treatment of lymphedema in head and neck cancer patients. ${ }^{11}$ Most findings in lymphedema research, mainly in breast cancer patients, have shown that early intervention is the best management and yields the best outcomes. As with other chronic conditions, early identification and timely, appropriate treatment of lymphedema is critical to improve clinical outcomes, to decrease symptom burden and functional impairment, and to improve overall quality of life in head and neck cancer patients. ${ }^{10}$

\section{Improving recognition and treatment}

Head and neck oncologic treatment is increasingly offered outside the network of specialist academic hospitals, at hospitals serving more localized communities where the neediest, sickest patient groups may be receiving less than optimal care. ${ }^{3}$ This challenges community hospitals to provide optimal treatment, similar to that being offered at nationally recognized institutions. In January 2012, we implemented a prehabilitation program in our community hospital cancer center to provide early intervention for our patients based on the understanding that proper and prompt treatment for patients with early signs of lymphedema should be a priority. ${ }^{12}$ In this article, we outline how we implemented the program and the describe improvements we observed before and after the implementation of the program.

\section{The prehabilitation program \\ The role of the nurse navigator}

Before the introduction of the prehabilitation program, our pattern of practice was to refer patients to oncology rehabilitation for lymphedema management after they had completed their medical treatment with surgery, radiation, and chemotherapy. In 2012, that was changed to a prehabilitation model of care that was overseen by a head and neck nurse navigator. This focus on prehabilitation begins with patients being referred to oncology rehabilitation at the time of cancer diagnosis for baseline assessment of head and neck swelling. In addition, there is assessment of the many possible other side effects associated with head and neck cancer and its treatment, namely loss of range of motion of the neck, jaw (trismus), and/or shoulders, postural deficits, functional loss, pain, balance dysfunction with fall risk, weakness, and fatigue. Therapeutic interventions are initiated as needed and appropriate. This process also raises awareness of a condition that has been described as under-recognized and under-treated. ${ }^{3}$

The nurse navigator sits in on each radiation oncology consultation and aids in "navigating" patients through their treatment. The nurse ensures that each patient is referred to different ancillary services from the outset, such as seeing a dietician, social worker, physical/occupational therapist and certified lymphedema therapist, speech pathologist, and financial assistance advisor, if necessary (Table 1).

\section{Assessment of lymphedema}

Measurement of head and neck lymphedema is a challenge. ${ }^{10}$ In our program, the physical therapy assessment also includes the evaluation of several other morbidities associated with head and neck cancer and its treatment,

$$
\begin{aligned}
& \text { TABLE } 1 \text { Prehabilitation assessments and preparationa } \\
& \text { Assessment of possible concomitant side effects } \\
& \text { - Loss of motion of the neck, jaw and shoulders } \\
& \text { - Functional lossb } \\
& \text { - Pain } \\
& \text { - Balance dysfunction with fall risk } \\
& \text { - Weakness } \\
& \text { - Fatigue } \\
& \text { Preparation } \\
& \text { - Education in basic lymphatic anatomy/ physiology } \\
& \text { and lymphedema risk } \\
& \text { - Home exercise program established if indicated } \\
& \text { - Plan for follow-up reassessment and/or treatment } \\
& \text { established }
\end{aligned}
$$

apatients are referred for prehabilitation at the time of cancer diagnosis, to provide a baseline assessment of head and neck swelling, and of various dysfunctions. bFunctional losses include deficiencies in self-care, sleeping, concentration, driving, reaching and lifting, ability to work or participation in recreational activities. 
such as range of motion, weakness, fatigue, radiation fibrosis, balance dysfunction, and risk of falling (Table 2).

Patient-reported outcomes are essential to fully capture observable and unobservable symptoms (eg, sensations) as well as the functional impacts of lymphedema. ${ }^{10}$ In addition to lymphedema, there are many other morbidities that may be assessed on the basis of patient-reported outcome tools, such as upper extremity function with QuickDASH. ${ }^{13}$ At our clinic for head and neck cancer patients we use the Neck Disability Index (NDI) ${ }^{14}$ and Care Connections $(\mathrm{CC})^{15}$ survey for the patient-reported outcomes. The Quick DASH, NDI, and CC tools all assess standard functional outcomes that are not specific to lymphedema, but are useful in documenting changes related to lymphedema. We initially used the CC survey and later transitioned to using the NDI. Neck pain is common with lymphedema in the head and neck region, and the NDI is a valid, reliable, responsive and internally

TABLE 2 Clinical evaluation of lymphedema in head and neck cancer patients

Patient-reported outcomes
Self-reporting of swelling symptoms
Neck pain
Other observable and non-observable symptoms
(such as sensations)
Quick Dash questionnaire (for upper-extremity
functional assessment)
Care Connections questionnaire
Neck Disability Index questionnaire
Clinician-reported outcomes
Physical examination to assess external lymphedema
Visual inspection, pitting or non-pitting edema,
tissue texture
Endoscopy to assess internal lymphedemaa
Functional testing
Range of musculoskeletal motion
Neck, jaw (trismus) and shoulders
Weakness and fatigue
Spinal accessory nerve palsy
Peripheral neuropathy (chemotherapy induced)
Balance dysfunction (with fall risk)
Radiation-induced fibrosis
Swallowing (dysphagia)
Technical (objective) measurements
Tape measurements

Tape measurements

Digital photography

-Not available at Disney Family Cancer Center. consistent clinical tool to measure self-reported disability in patients with neck pain. ${ }^{16}$ These questionnaires were completed by the patients at their initial assessment, at reassessment, and at time of discharge.

Although objective criteria for external lymphedema have not been established, simple measurements such as using a tape measure to record neck circumference, allow a useful longitudinal assessment. Digital photography may be effective in the documentation and subjective evaluation of changes of external lymphedema. ${ }^{10,17}$ However, there are some limitations with photography because although external photographs (including digital photography and three-dimensional imaging) can capture some features, such as changes in contours, symmetry, and changes in skin quality and color, they do not detect changes in skin and soft tissue texture and compliance (Table 3). ${ }^{10}$

\section{Impact on clinical outcomes}

We retrospectively reviewed the medical records of 230 head and neck cancer patients who had been treated at our center between June 2008 and June 2015. Complete clinical data were available for 190 patients. The following information was extracted from each patient's chart: whether they developed lymphedema, tumor stage, had surgery, radiation dose, type of chemotherapy given, their smoking history, if they had had a neck dissection and the primary site of the tumor (Table 3).

Incidence in different time periods. Of the 190 patients with complete records 78 (41\%) were found to have lymphedema. These were all patients undergoing treatment for head and neck cancer during June 2008-June 2015. The prehabilitation program was initiated with the hiring of a nurse navigator for head and neck cancer, starting in January 2012. It is interesting to note that the incidence of lymphedema was $27 \%$ before the program was started, but after nurse navigator joined the team, the incidence increased significantly to $48 \%(P=.0002)$, in line with published expectations. This increase in recorded incidence may be attributable to the greater awareness of lymphedema intentionally fostered by the prehabilitation program.

Smoking history. Patients' lifetime smoking history was retrieved from their medical records, based on their verbal admission of tobacco use. Most of the patients $(n=110)$ self-reported a history of smoking. Of those with a history of smoking, 36 (33\%) developed external lymphedema after treatment for head and neck cancer, and 74 (67\%) did not. However, this difference was not statistically significant. Hence, although smoking is a risk factor for head and neck cancer, it was not associated with the development of external lymphedema in our cohort of patients. 
TABLE 3 Clinical observations

\begin{tabular}{|c|c|c|c|c|}
\hline \multirow[b]{2}{*}{ Parameter } & \multicolumn{4}{|c|}{ Lymphedema status, n (\%) } \\
\hline & $\begin{array}{l}\text { No. of } \\
\text { patients (\%) }\end{array}$ & With & Without & Significance \\
\hline \multicolumn{5}{|l|}{ Period } \\
\hline June 2008- June 2015 & $190(100)$ & $78(41)$ & $112(59)$ & NS \\
\hline June 2008- December 2011 & $62(33)$ & $17(27)$ & $45(73)$ & NS \\
\hline January 2012- June 2015 & $128(67)$ & $61(48)$ & $67(52)$ & $P=.0002^{1}$ \\
\hline Self-reported lifetime smoking history & $110(58)$ & $36(33)$ & $74(67)$ & NS \\
\hline Squamous-cell carcinoma & $156(82)$ & $70(45)$ & $86(55)$ & NS \\
\hline Tumor stage 3 or 4 & $121(64)$ & $51(42)$ & $70(58)$ & NS \\
\hline \multicolumn{5}{|l|}{ Position of tumor } \\
\hline Oral cavity ${ }^{a}$ & $26(14)$ & $12(46)$ & $14(54)$ & NS \\
\hline Pharynx & $111(58)$ & $50(45)$ & $61(55)$ & NS \\
\hline Nasopharynx & $13(7)$ & $1(8)$ & $12(92)$ & $P=.017^{1}$ \\
\hline Oropharynx & $87(46)$ & $47(60)$ & $40(36)$ & $P=.044^{1}$ \\
\hline Base of tongue & $45(24)$ & $25(56)$ & $20(44)$ & NS \\
\hline Tonsil & $38(20)$ & $20(53)$ & $18(47)$ & NS \\
\hline Other & $4(2)$ & $2(50)$ & $2(50)$ & NS \\
\hline Hypopharynx & $11(6)$ & $2(18)$ & $9(82)$ & $P=.04^{1}$ \\
\hline Larynx & $2(1)$ & $1(50)$ & $1(50)$ & NS \\
\hline Parotid gland & $14(7)$ & $1(7)$ & $13(93)$ & $P=.012^{1}$ \\
\hline Other & 37 (19) & $14(38)$ & $23(62)$ & NS \\
\hline \multicolumn{5}{|l|}{ Treatment } \\
\hline No resection or neck dissection ${ }^{b}$ & $95(50)$ & $25(26)$ & $70(74)$ & $P=.015^{1}$ \\
\hline Resection of primary tumor ${ }^{b}$ & $65(34)$ & $35(54)$ & $30(46)$ & $P=.0004^{2}$ \\
\hline Resection plus neck dissection ${ }^{b}$ & $26(14)$ & $18(69)$ & $8(31)$ & $<.0001^{2}$ \\
\hline Neck dissection only ${ }^{b}$ & $4(2)$ & $4(100)$ & $0(0)$ & $P=.006^{2}$ \\
\hline Radiation, no surgery ${ }^{b}$ & $121(64)$ & $43(36)$ & $78(64)$ & NS \\
\hline Radiation plus surgery ${ }^{b}$ & $69(36)$ & $35(51)$ & $34(49)$ & $P=.04^{3}$ \\
\hline Radiation $<60$ cGy & $28(15)$ & $7(25)$ & $21(75)$ & NS \\
\hline Radiation 60-69.6 cGy & $55(29)$ & $24(44)$ & $31(56)$ & NS \\
\hline Radiation $>70$ cGy & $105(55)$ & $45(43)$ & $60(57)$ & NS \\
\hline Radiation dose unknown & $2(1)$ & $2(100)$ & $0(0)$ & NS \\
\hline Chemotherapy & 131 (69) & $58(44)$ & $73(56)$ & NS \\
\hline
\end{tabular}

Significance determined using Pearson chi-square test of association. NS means not statistically significant (significant $=P<.05$ ). The groups compared are shown in the superscripts: $P 1$ values are comparisons with the whole cohort (2008-2015); $P 2$ values are in comparison with the 'no dissection or resection' patients; and the P3 value is a comparison with the 'radiation no surgery' patients.

aral cavity includes the oral tongue, buccal mucosa, retromolar trigone, and floor of the mouth. bThe term surgery includes both resection of the primary tumor and neck dissection, the term resection refers to resection of the primary tumor.

\section{Type of tumor}

Most of the patients ( $\mathrm{n}=156,82 \%)$ had squamous cell carcinomas (SCC). Of those, $45 \%$ developed external lymphedema and 55\% did not. Therefore, having SCC did not predispose to lymphedema. The other cancers were mixed type, mainly adenocaricoma, but their numbers were too small to draw statistical conclusions. 


\section{Stage of the tumor}

About two thirds of the patients $(n=121,64 \%)$ had stage 3 or 4 cancer. However, treatment of more advanced cancers was not associated with lymphedema development.

\section{Site of the tumor}

The literature suggests that patients with a primary tumor in the throat are at increased risk for lymphedema. ${ }^{5}$ The American Cancer Society has defined cancers of the oropharynx (throat) as including the base of the tongue (back third of the tongue), the soft palate, the tonsils, and the side and back walls of the throat. ${ }^{18}$ In our head and neck cancer cohort, patients with primary tumors of the oropharnyx were, perhaps, more susceptible to lymphedema $(P=.044$, Table 3). By contrast, in our cohort of patients, those with nasopharyngeal, hypopharyngeal, and parotid gland tumors were significantly less likely to develop lymphedema $\left(P_{\mathrm{S}}=\right.$ $.017, .04, .012$, respectively).

\section{No surgery}

Half of our patients $(n=95)$ were not treated with surgery. In the patients who did not have surgery, 25 (26\%) developed lymphedema, whereas 70 (74\%) did not. Hence, although the incidence of lymphedema was significantly lower in patients who did not have surgery $(P=.015)$, lymphedema did develop in patients who did not have a surgical procedure.

\section{Resection of primary tumor without neck dissection}

Of the 64 patients who had surgery, but without neck dissection, 35 (55\%) developed external lymphedema. Compared with the no-surgery patients, the doubling of the incidence (from $26 \%$ to $55 \%$ ) was highly significant $(P=.0004)$. These findings are compatible with the literature reports that surgery increases the incidence of lymphedema, which is not surprising because surgery and subsequent scarring is known to compromise the lymphatic system.

\section{Resection of primary tumor with neck dissection}

The incidence of external lymphedema was increased to $69 \%$ when patients were subjected to both surgery and neck dissection. Compared with the June 2008-June 2015 cohort, there was a significant increase in the incidence of lymphedema in the neck dissection group $(P=.007)$. Neck dissection involves the removal of lymph nodes and disruption of the lymphatic vessels, so it is not surprising that there is a higher incidence of external lymphedema. In our practice, neck dissections increased in frequency every year from June 2008 until December 2011, when 8 patients underwent neck dissections, 6 (75\%) of whom developed lymphedema. Since January 2012, when the prehabilitation program was implemented, the number of neck dissections have declined, with more patients receiving chemoradia- tion and surgery being reserved for surgery. Hamoir and colleagues have reported that neck dissection is no longer justified unless there is clinically residual disease in the neck. $^{19}$

\section{Radiation}

Lymphedema occurred in patients regardless of the dose of radiation received. Although the incidence of lymphedema seemed to be higher in patients who received more than 60 cGy, that difference was not statistically significant (Table 3). We had expected a relationship between radiation damage and greater lymphedema, but that was not evident in our patients.

\section{Chemotherapy}

The majority of patients $(n=131,69 \%)$ received chemotherapy. The exposure to chemotherapy was not correlated with the risk of external lymphedema in our cohort of patients, with 58 of the 131 treated patients (44\%) developing lymphedema, compared with $73(56 \%)$ of treated patients who did not (Table 3).

\section{Complete decongestive therapy}

All patients with documented lymphedema were evaluated for complete decongestive therapy (CDT). Contraindications to CDT included congestive heart failure, renal failure, acute infection, peripheral artery disease, upper-quadrant deep vein thrombosis, and carotid artery stenosis. Eligible patients were referred to a certified lymphedema therapist for CDT. As the program evolved, patients at risk for lymphedema were referred for CDT early on, usually at the time of diagnosis, to improve early identification and surveillance of lymphedema.

CDT included manual lymph drainage, compression bandaging (Figure), decongestive exercises, skin care, and education in swelling self-management. Manual lymph drainage is a specialized light pressure hands-on technique that reduces swelling by enhancing lymphatic reabsorption and flow. Compressive bandaging/garments increase venous and lymphatic drainage and soften fibrotic tissue. Continued use of compression depends on progress. In head and neck cancer patients, the need for lifelong compression is not evident when they are treated early and there is good patient compliance. ${ }^{8}$ Therapeutic exercise enhances lymphatic and venous circulation, and good skin care reduces the risk of infection.

Patients' responses to CDT were documented with digital photographs that were taken at each visit and, more recently, use of the NDI.

\section{Communication and education}

The head and neck cancer nurse navigator attends the cancer center's multidisciplinary head and neck tumor board, which has representation from otolaryngology, diagnostic 


\section{How We Do It}

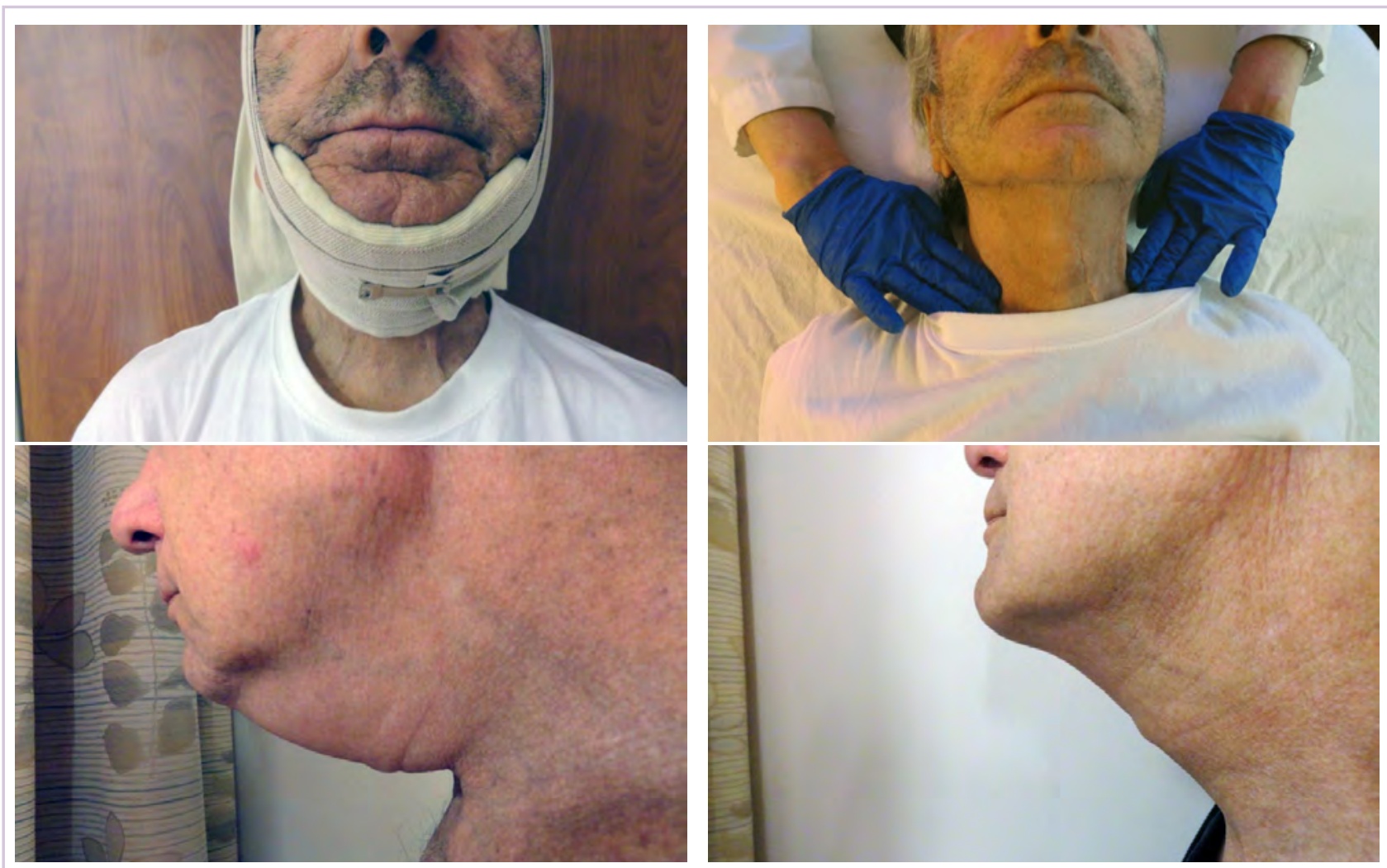

FIGURE Compression and manual lymph drainage in head and neck cancer. A, Compressive bandages and garments are designed to reduce fluid content of tissues. B, Specialized light manual techniques improve lymph flow and reduce lymphedema. C, A patient who developed lymphedema after tonsillectomy for right tonsillar cancer, before complete decongestive therapy. D, The same patient at discharge, after 11 visits for CDT over 4 months.

radiology, pathology, radiation oncology, medical oncology, reconstructive surgery, oncology rehabilitation (physical/ occupational therapist), dietary services, speech pathology, social services and clinical research. This regular contact allows for earlier awareness about which patients are at greater risk for developing lymphedema, thus enabling early intervention (and patient education) in a timely manner.

Education of the patient, before cancer therapy, of the risks of lymphedema is very important. Before the implementation of the prehabilitation program, some patients did not fully comprehend what a painful and debilitating consequence of cancer treatment lymphedema could be.

\section{Discussion}

We introduced a prehabilitation program to detect and treat lymphedema in head and neck cancer patients in January 2012 part way through following an observation cohort from June2008 through June2015. Central to this, in our center, was the appointment of a nurse navigator whose primary focus was on head and neck cancer patients. We placed a high priority on the early detection and treatment of lymphedema because do so has been associated with better outcomes in other centers.
One immediate consequence of the inception of our program was the identification of more patients with external lymphedema. Our detected incidence rose significantly $(P=.0002)$, from $27 \%$ in the period June 2008-December 20112010, before the program, to 48\% during the January 2012-June 2015 period, after the inception of the program. This later incidence rate is in line with published incidence rates in most centers. However, it is still somewhat short of the $75 \%$ suggested in one center, ${ }^{9}$ which suggests we are either we are underdetecting lymphedema or there are differences in definition criteria or sensitivity levels for defining lymphedema.

There are currently no specific objective measures of lymphedema, so there is bound to be some variation in diagnosis rates. In our program, we rely heavily on the patient-reported outcome measures, the NDI instrument, and digital photography to detect and monitor lymphedema, starting with the pretreatment baseline values that are established for each patient.

The use of digital photography in our community hospital setting, which includes taking photographs before and after treatment and at each visit, motivates and encourages patients and provides a tool for clinical lymphedema thera- 
pists to visually document benefits of treatment. Patients' motivation and compliance with their established home program for head and neck lymphedema self-management are essential. The elements of the home program may include self-manual lymph drainage, home-modified compression bandaging and garment wear, therapeutic exercises, and skin care. Patients with lymphedema who adhered closely with their therapy program were more than 8 times more likely to improve compared with noncompliant patients. ${ }^{17}$

Some groups of patients have a greater risk of developing lymphedema than others, ${ }^{5}$ so the development of an algorithm to predict lymphedema seemed possible. However, in our cohort of patients, only neck dissection, with its disruption of the lymphatic system of the neck, was strongly associated with external lymphedema (Table 3). It is important to note that some patients who did not undergo surgery developed lymphedema. In our patients, high doses of radiation alone did not seem to predispose to lymphedema. That suggests that no group of head and neck cancer patients should be ignored, which is why we did routine screening of all patients before, during, and after treatment.

Our protocol falls short in the detection of internal lymphedema. For example, information on swallowing gathered by our speech pathologists (in a different department) has not, so far, been included in our assessment. This is one opportunity to improve on our approach, especially because speech difficulties may be associated with internal lymphedema. In addition, we are not equipped for the requisite internal examinations. Unfortunately, there are no practical and successful treatments for patients suffering from internal swelling. This represents a challenge for the medical community to better meet this need. Therefore, although we are missing some assessments of internal lymphedema, this is of little therapeutic consequence at this time.

The increase in the detected incidence of external lymphedema points to a practice gap that has been resolved by the appointment of a dedicated nurse navigator who attends oncology reviews to share knowledge and information. Another educational effort has been made with the patients themselves to increase compliance and improve continuous care at home.

There is always room for improvement, however, either by feedback acquired from other institutions and hospitals or through the future introduction of more objective assessment techniques.

\section{Conclusions}

The introduction of the prehabilitation program at our center has coincided with a significantly improved detection rate for external lymphedema in head and neck cancer patients. It may be because the program emphasizes education about lymphedema that awareness of the condition has increased throughout the center. It is now widely recognized that all patients are at risk of lymphedema regardless of whether they fall into an acknowledged high-risk group. Our experience shows that there is no significant difference between treatment modalities apart from neck dissection. In our population, the use of this procedure is decreasing. External lymphedema can develop even in patients who do not have surgery. Therefore, there is no sound way to predict which patients are most likely to suffer from the accumulation of fluid in their head and neck after treatment for head and neck cancer. Thus, an assessment as described here, during and after treatment for all patients, is warranted. Patients are now being seen earlier as a part of the prehabilitation program, which facilitates access to complete decongestive treatment at an earlier stage, improves patient outcomes, and increases patient satisfaction with their treatment. Our prehabilitation program could serve as a model for other community hospital centers in achieving outcomes that are as good as those in academic centers.

\section{Acknowledgments}

The authors thank Irene Kadota and Heather Peters, from the Department of Radiation Oncology, and Julianne Courtenay, from the Department of Physical Therapy at the Disney Family Cancer Center, Burbank, California, for providing the original clinical data for analysis.

\section{References}

1. The National Lymphedema Medical Advisory Committee. The diagnosis and treatment of lymphedema. National Lymphedema Network. http://www.lymphnet.org/pdfDocs/nlntreatment.pdf. Updated February 2011. Accessed April 26, 2017.

2. McGarvey AC, Osmotherly PG, Hoffman GR, Chiarelli PE. Lymphedema following treatment for head and neck cancer: impact on patients, and beliefs of health professionals. Eur J Cancer Care (Engl). 2014;23(3):317-327.

3. Bhattacharyya N, Abemayor E. Patterns of hospital utilization for head and neck cancer care: changing demographics. JAMA Otolaryngol Head Neck Surg. 2015;141(4):307-312.

4. Deng J, Ridner SH, Dietrich MS, et al. Prevalence of secondary lymphedema in patients with head and neck cancer.J Pain Symptom Manage. 2012;43(2):244-252.

5. Deng J, Ridner SH, Dietrich MS, et al. Factors associated with external and internal lymphedema in patients with head-and-neck cancer.

Int J Radiat Oncol Biol Phys. 2012;84(3):e319-328.

6. Flores AM, Spinelli BA, Eden MM, Galantino ML. EDGE task force on head and neck cancer outcomes: a systematic review of outcomes measures for quantifying external lymphedema. Rehabil Oncol. 2015;33(2):15-23.

7. Ridner SH, Doersam J, Galford E. An update on lymphedema of the head and neck. http://www.lymphnet.org/pdfDocs/Vol_28-N2 Update_HN.pdf. Published April-June 2015. Accessed April 26, 2017.

8. Smith BG, Hutcheson KA, Little LG, et al. Lymphedema outcomes in patients with head and neck cancer. Otolaryngol Head Neck Surg. 2015;152(2);284-291.

9. Naqvi SHS, Karni RJ, Tan IC, et al. Int J Rad Oncol Biol Phys. 2016;4:927-928.

10. Deng J, Ridner SH, Aulino JM, Murphy BA. Assessment and measurement of head and neck lymphedema: state-of-the-science and 


\section{How We Do It}

future directions. Oral Oncol. 2015; 51(5):431-437.

11. Purcell A. Head and neck lymphedema management practices. J Lymphedema. 2013;8(2):8-15.

12. Paskett ED, Dean JA, Oliveri JM, Harrop JP. Cancer-related lymphedema risk factors, diagnosis treatment and impact: a review. J Clinl Oncol. 2012;30(30):3726-3733.

13. Quick DASH questionnaire. http://www.dash.iwh.on.ca/aboutquickdash. [Last update not stated.] Accessed May 18, 2017.

14. Neck Disability Index questionnaire. www.aaos.org/uploadedFiles/ NDI.pdf Accessed May 18, 2017.

15. Care Connections questionnaire. http://www.careconnections.com/. Accessed May 18, 2017.

16. Galantino ML, Eden MM, Spinelli BA, Flores AM. EDGE task force on head and neck cancer outcomes a systematic review of outcome measures for temporomandibular-related dysfunction. Rehabil Oncol. 2015;33(1):6-14.

17. Deng J, Ridner SH, Murphy BA. Lymphedema in patients with head and neck cancer. Oncol Nurs Forum. 2011;38(1):e1-e10.

18. What are oral cavity and oropharyngeal cancers? American Cancer Society. http://www.cancer.org/cancer/oralcavityandoropharyngealcancer/detailedguide/oral-cavity-and-oropharyngeal-cancer-whatis-oral-cavity-cancer. Last revised August 8, 2016. Accessed April 26, 2017.

19. Hamoir M, Schmitz S, Gregoire V. The role of neck dissection in squamous cell carcinoma of the head and neck. Curr Treat Options Oncol. 2014;15:611-624. 